\title{
Dynamics and Prediction of Clicks on News from Twitter
}

\author{
Arthi Ramachandran \\ University of Chicago \\ Chicago, USA \\ arthir@cs.columbia.edu
}

\author{
Lucy Wang \\ Buzzfeed \\ New York, USA \\ lucyxw1@gmail.com
}

\author{
Augustin Chaintreau \\ Columbia University \\ New York, USA \\ augustin@cs.columbia.edu
}

\begin{abstract}
Social networks are a major gateway to access news content. It is estimated that a third of all web visits originate on social media, and about half of users rely on those to keep up-to-date with world events. Strangely, no model has been proposed and validated to study how to reproduce and interpolate clicks created by social media. Here we study news posted on Twitter, leveraging public information as well as private data from a popular online publisher. We propose and validate a simple two-step model of information diffusion that can be easily interpreted and applied using only public information to determine current and future clicks.
\end{abstract}

\section{ACM Reference Format:}

Arthi Ramachandran, Lucy Wang, and Augustin Chaintreau. 2018. Dynamics and Prediction of Clicks on News from Twitter. In HT '18: 29th ACM Conference on Hypertext and Social Media, July 9-12, 2018, Baltimore, MD, USA. ACM, New York, NY, USA, 5 pages. https://doi.org/10.1145/3209542.3209568

\section{INTRODUCTION}

Data from social networks is being used to predict a range of outcomes, including the performance of various stocks [5], the progress of various epidemics [24], statistics on crimes [28], and the box office success of movies [3,29]. However, little is known on how links distributed on social media generate clicks even though social media based traffic is a massive source of web traffic.

Among links exchanged on social media, predicting the attention gathered by links to news articles seems especially important. An increasing fraction of the population receives their news via social media posts. Further, that evolution of news distribution has raised concerns about its societal impact, including the formation of echo chambers or the spread of dishonest information. Without a satisfactory estimation of clicks, that impact is difficult to gauge. We may be ignoring a silent majority of readers who devote attention only through clicking on, but not sharing or posting content. For these reasons, we urgently need methods that interpolate attention measured in clicks using data currently available to social scientists such as shares and followers popularity.

Estimating clicks is hard due to lack of publicly available data and the complex interplay of several factors pertaining to the content,

This material is based upon work supported by the National Science Foundation under Grant No.1254035 and 1514437; A. Ramachandran conducted it at Columbia University.

Permission to make digital or hard copies of all or part of this work for personal or classroom use is granted without fee provided that copies are not made or distributed for profit or commercial advantage and that copies bear this notice and the full citation on the first page. Copyrights for components of this work owned by others than ACM must be honored. Abstracting with credit is permitted. To copy otherwise, or republish, to post on servers or to redistribute to lists, requires prior specific permission and/or a fee. Request permissions from permissions@acm.org.

HT '18, fuly 9-12, 2018, Baltimore, MD, USA

(C) 2018 Association for Computing Machinery.

ACM ISBN 978-1-4503-5427-1/18/07 . \$15.00

https://doi.org/10.1145/3209542.3209568 source, and time. In this paper, we leverage a unique data set and modeling design to model observed click dynamics:

- We analyze impression dynamics on Twitter. (Section 3)

- We justify a two-step, memoryless generative model of link clicks on Twitter, with just a few time-invariant parameters. (Section 4)

- We can then use those lessons learned to interpolate the (private) clicks from (public) social media activity either applied in real time or in foresight. (Section 5)

Our analysis reveals important insights: In contrast with share cascade dynamics, click evolution naturally unfolds at a slower pace. As a result, we demonstrate much higher accuracy in foresight. This indicates that the opportunities to predict web traffic may be underestimated in previous analyses.

\section{A SURVEY OF CLICKS ESTIMATION}

We feel the need to clarify how interpolating clicks differs from previous works and fits into the large body of work studying the dynamics of news diffusion on Twitter. Information diffusion has been studied to quantify influence $[4,7]$ and how social networks affect news sharing $[1,2]$. Our results are a first step to analyze the behavior of the large majority of silent social media participants, using clicks as a proxy for their collective reading habits. Historically, most models of clicks have been designed for online advertising $[10,19]$ which is a different context from social media.

The vast majority of work studying information diffusion measure popularity through posting activity (shares, tweets, retweets). Some proposed models exploit microscopic information about the earliest posts (such as detailed time statistics, or network structure) $[9,18]$. Clicks are more sensitive and hence can almost never be observed at such microscopic individual level. Other models of posting dynamics include using a Bayesian approach [30], reinforcing processes $[25,31]$, or dynamical process under external influence [20]. But while posts appear as bursts in a short-time frame, clicks appear and decay at larger time scales [11].

Finally, a handful of works have addressed the prediction of web traffic and page views online, but are radically different. Studies on YouTube videos, which publicly disclose the view counts, use the popularity observed in the first hour alone ([26]), aggregate retweet counts from subsequent hours ([21]) and explicitly model the impact of social media posts as a decaying function ([23]). Recently, [15] posed the prediction of page views for news articles after a day as a regression task. Like [26], it confirmed that (among many features) the early page views observed after $30 \mathrm{mn}$ has the highest correlation. We cannot compare our results directly as this paper offers no reproducible algorithm or model and contains no result applied outside of a proprietary dataset. Our results suggest that a much stronger predictive value can be extracted from Twitter. 


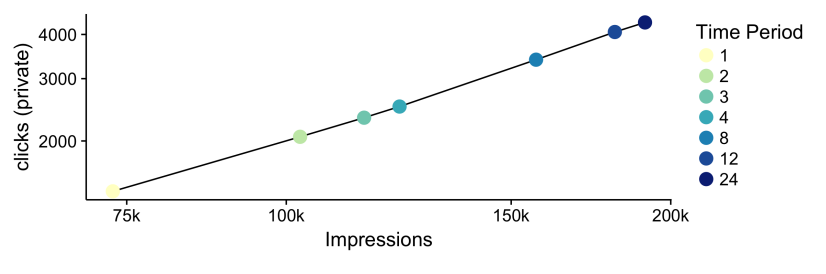

Figure 1: Evolutions of clicks with age for an example link.

\section{FOLLOWERS, IMPRESSIONS AND CLICKS}

To reproduce click dynamics, we conduct the first complete analysis of news diffusion on Twitter across all three stages of diffusion: tweets, clicks, and the intermediate step of impressions.

\subsection{News Media and Publisher Datasets}

We start out with BuzzFeed's internal (private) Twitter dataset which has the hourly metrics on each BuzzFeed tweet. We use this source of truth to validate our estimates from public data. The first public data source gives us follower counts of tweets (to estimate impressions i.e., the number of times a link appeared on anyone's screen). The second public source allows us to estimate clicks.

Collecting the Source of Truth We use a proprietary content publisher dataset from BuzzFeed. This data has hourly link clicks, retweets, and impressions for more than 40 active Twitter accounts. The readership information provided by this dataset is used solely here for the purpose of validating a reproducible model. Since the content publisher data set contains proprietary information of a commercial nature, it cannot be disclosed in its entirety.

Collecting (Re)Tweets and Followers We used Twitter's REST API to scrape all tweets and subsequent retweets by BuzzFeed accounts for August 3 to 17, 2016 (4K tweets). Each (re)tweet provides the publicly available follower count of the (re)tweeting user, from which we estimate potential readership, and therefore impressions. This data ignores private retweets, which make up a trivial fraction of retweets [27].

Collecting Impressions and Clicks For each of the Buzzfeed tweets collected, we used bit.ly's API to gather all link clicks referred from Twitter (those with twitter. com or t. co as the listed referrer domain). We collected data for the periods of hours $1,2,3,4,5-$ $8,9-12,13-24$. We considered only links with bit.ly URLs which completed a 24 -hour lifecycle within the date range (1.4K tweets).

We validate our analyses with a data set that includes five major news outlet (Multiple News Media ${ }^{1}$ ).

\subsection{Click evolution and its metrics}

Receptions, impressions, and distinct click ratios When a Twitter user (re)tweets a link, their followers join the set of "receiving" Twitter users who are susceptible to seeing the link. We call that event a reception. The number of receptions of a link is the sum of follower counts for all accounts that (re)tweeted the link. Previous work has shown that overestimation from double-counting followers is less than $20 \%$ for $75 \%$ of reception counts [12]. ${ }^{2}$

\footnotetext{
${ }_{1}$ available at http://www-sop.inria.fr/members/Arnaud.Legout/Projects/sotweet.htm ${ }^{2}$ While one can theoretically compute the number of unique accounts in the "receiving set" to remove multiplicity, the number of API queries involved quickly makes this prohibitively expensive.
}
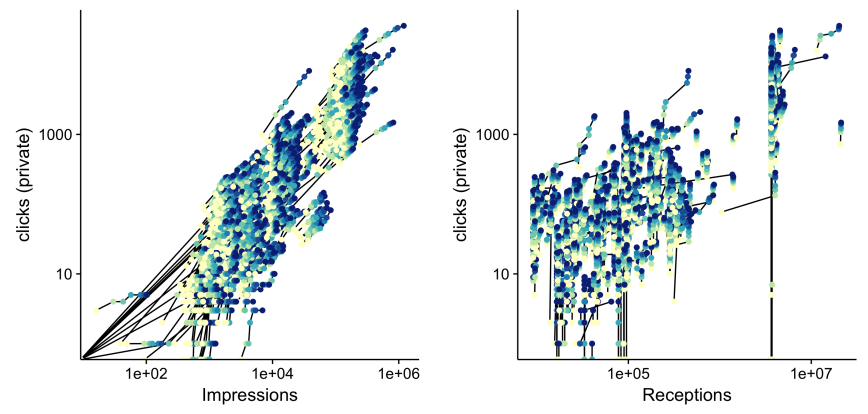

Figure 2: Evolutions of clicks with age ( $y$-axis) (left) by impressions ( $x$-axis) for all links in Content Publisher (right) by receptions ( $x$-axis) where no simple dependence emerges.

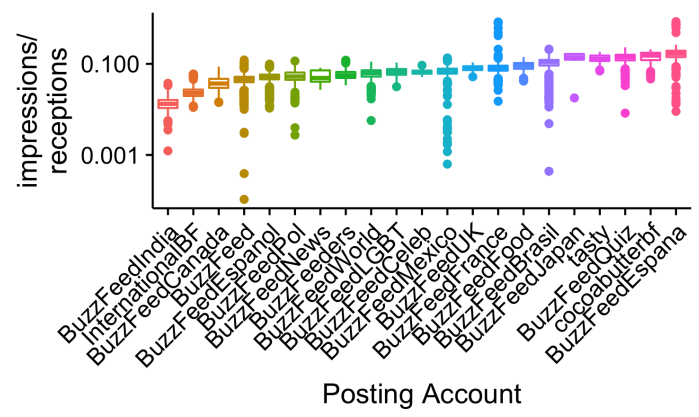

Figure 3: Impressions to Receptions Ratio, seen for all URLs across different accounts, as estimated after $24 \mathrm{~h}$.

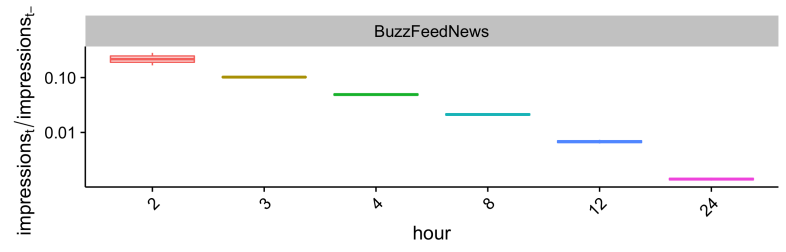

Figure 4: Hourly impressions produced decreases geometrically with time

Most prior studies of online traffic focus on Clicks Per Impression (equivalently Click Through Rate). However, the number of impressions is almost never available when studying social media. We use the following:

- Clicks Per Impressions (CPI): $\frac{\# \text { clicks }}{\text { \# impressions }}$

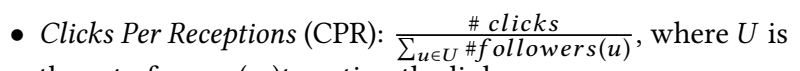
the set of users (re)tweeting the link.

Receptions and Impressions have distinct dynamics The dynamics of receptions and impressions in click generation show substantial differences (Figure 2). Here, each link is a line connecting dots that represent that same link at different time, so that data for a given link becomes darker with its age (example in Figure 1). 


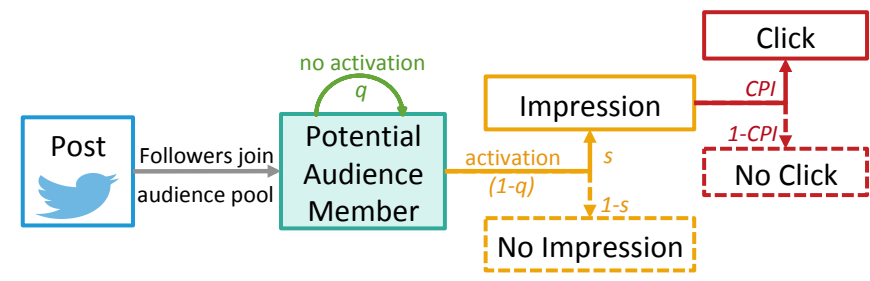

Figure 5: Model of evolution of a click from an initial post to an impression to a click.

The line relating impressions and clicks at different times is, in log-scale, almost perfectly linear. Moreover, that linear trajectory is exhibited by almost all links in the data, regardless of how many clicks it starts out with (Figure 2 left). This indicates that up to a multiplying constant, clicks and impressions per link follow a linear dependence. We conclude that clicks can be derived from a link's impression by a simple multiplication and the multiplying factor is invariant so it can be estimated once. In contrast, we find that with the number of receptions of a news link (i.e., the sum of followers of all posting sources), the effects of age do not follow a simple dependence (Figure 2 right).

A different model is needed So far our results explain the failure of most click interpolation methods: Receptions are not sufficient alone to predict clicks, while impressions would be but are almost never available. Our results point to a possible new design: studying the dynamics creating impressions from receptions, with the hope to build a robust estimation of (private) impressions using (public) receptions with other available factors that play a role.

\subsection{In depth dynamics of Twitter diffusion}

With a diverse dataset from multiple content sources we can isolate the role of different factors on impression generation. The ratio of impressions divided by receptions across all links varies a lot (Figure 3). But strikingly, almost all this ratio's variance comes from the content source and within the source there is little variation. That result essentially confirms a posteriori that impressions are created based on how the source's particular followers pay attention to Twitter, somewhat independently of the specific content of a link.

In Figure 4, the hourly impressions produced by links from the Buzzfeed news account, normalized by impressions produced in the first period, are shown to follow a geometric decreasing trend with few variations. Similar trends have been observed for all accounts and are omitted due to space constraints. Those observations allow us to design an interpretable generative model of clicks on Twitter.

\section{CLICK GENERATING MODEL}

We model click and impression generation over time as summarized in Figure 5. The only variable for each link is the size of the potential audience, or receptions (light green box). Each time step represents a unit of time (e.g., an hour). At the end of each time step, we first increase the potential audience by the sum of followers for any tweet or retweets sent during that hour containing the link. Each audience member independently then decides to "activate" an impression with a fixed probability (solid orange arrow) or not

\begin{tabular}{ll}
\hline \hline Variable & Description \\
\hline \hline$x_{t}$ & observed cumulative receptions in time $t$ \\
\hline$y_{t}$ & actual cumulative impressions at time $t$ \\
$\tilde{y}_{t}$ & predicted cumulative impressions at time $t$ \\
\hline$z_{t}$ & actual cumulative clicks at time $t$ \\
$\tilde{z}_{t}$ & predicted cumulative clicks at time $t$ \\
$z_{t}^{b i t l y}$ & observed cumulative clicks from bit.ly at time $t$ \\
\hline$s$ & scaling parameter \\
$q$ & activation parameter \\
\hline
\end{tabular}

Table 1: Variables and Parameters of Model.

(dotted orange arrow). When a member activates, it is removed from the audience and follows the orange path. Otherwise, it remains in the potential audience as shown by the green loop. Of those who activate the impression, each independently decides to click on the article with a fixed probability (red solid box). Potential audience members leave as they activate while more members get added with new retweets. The model stops when the potential audience has no members left. Note that once a user is in the audience pool, it is no longer relevant when they entered the pool. Therefore, the dynamics of that model exhibit a memoryless property, typical of the geometric decay we observed before.

From Receptions to Impressions Formally, in this simple memoryless model, a fraction $1-q$ of the receptions are activated in each of the $T$ time periods (which corresponds to logging on to Twitter). Following that, all activated impressions are removed, and the process repeats on the remaining fraction $q$ in the next time period, and so on until none are left. Of all activated receptions in a given time slot, we assume a fraction $s$ will create an impression and the remainder will miss the link entirely. The form of decay in the potential audience pool size is a geometric decay and thus our model is a geometric memoryless model. Table 1 has a description of all the parameters and variables used. Let $x_{t}$ be the observed number of receptions in time $t \in[1, T]$ and $\tilde{y}_{t}$ be the predicted cumulative number of impressions in time $t$. Then we have $\tilde{y}_{t}=s \cdot \sum_{\tau=1}^{t} x_{\tau} \cdot\left(1-q^{t-\tau+1}\right)$.

From Impressions to Clicks The memory-less model above predicts the degree of exposure or potential audience to a given article. As previously observed, the ratio of clicks per impression remains stable over time. We can use a simple multiplicative factor to estimate the clicks from the predicted impressions at any hour $t$ $\left(\tilde{y}_{t}\right)$. This factor is computed at the first time period as the clicksto-impressions ratio at time $t=1$ i.e., $C P I_{t=1}=\frac{z_{1}}{\tilde{y}_{1}}$ where $z_{t}$ is the observed number of clicks at time $t$. Note that in cases where the private click information is not available, we can use the clicks obtained from the bit.ly API. Let $\tilde{z}_{t}$ be the predicted cumulative number of clicks at time $t$. Then $\tilde{z}_{t}=C P I_{t=1} \cdot \tilde{y}_{t}$.

Parameter Optimization For each article, we can study and learn its model parameters $s$ and $q$ minimizing the square error loss between the predicted number of clicks $\left(\tilde{y}_{t} \forall t \in[1, T]\right)$ and the actual number of clicks $\left(y_{t} \forall t \in[1, T]\right): \min _{s, q} J=\frac{1}{2} \sum_{\tau=1}^{T}\left(y_{\tau}-\tilde{y}_{\tau}\right)^{2}$. We used the L-BFGS-B optimization method [17] from Python's SciPy toolkit [14] for the minimization. The parameters learned for all links span a small range of values (the 5th percentile-95th percentile range for $s$ is [0.046, 0.103] and for $q$ is [0.207, 0.390]). 


\begin{tabular}{llcc}
\hline Method & Training & MAPE & MAPE (adjusted) \\
\hline Geom. & Hour 1 & $\mathbf{1 7 . 2 9} \%$ & $\mathbf{1 6 . 2 0} \%$ \\
HIP & Hour 1 & $102.2 \%$ & $99.5 \%$ \\
HIP (rec) & Hour 1 & $102.2 \%$ & $106.3 \%$ \\
Geom. & Hour 1, 2, 3, 4 & $\mathbf{1 2 . 6 8} \%$ & $\mathbf{1 1 . 9 7 \%}$ \\
HIP & Hour 1, 2, 3, 4 & $91.7 \%$ & $89.7 \%$ \\
HIP (rec) & Hour 1, 2, 3, 4 & $24.0 \%$ & $32.2 \%$ \\
\hline
\end{tabular}

Table 2: Comparing prediction accuracy of our method and HIP for estimates at $24 \mathrm{~h}$ for various training periods.

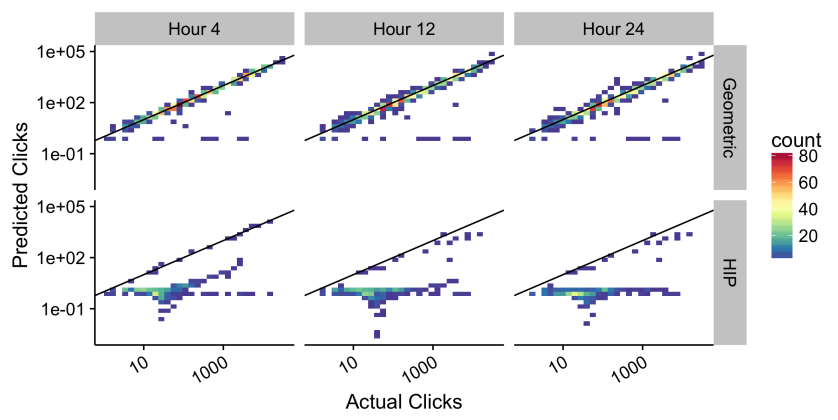

Figure 6: Prediction accuracy of our method and HIP with actual ( $x$-axis) vs predicted ( $y$-axis) values of clicks at different estimation times (shown for $4 \mathrm{~h}$ of training)

\section{REAL-TIME INTERPOLATION}

Since the parameters we've learned fall within a narrow range for all links, these parameter values can be used to interpolate hourly clicks for most links. For this study, we assume that in a short period (the training period, which can be up to $4 \mathrm{~h}$ here), a model has access to all previous clicks and impressions to estimate its own parameters (similar to [23, 25, 32]). After that period is over, the model only has access to the (public) process of posts and retweets and aims to to estimate clicks based on that activity as it unfolds.

Comparing with state-of-the-art When our model is trained on just 1 hour of retweets and clicks, it can interpolate each link's 24-hour click counts with mean absolute percentage error (MAPE) of $17.29 \%$ (and $16.20 \%$ in adjusted MAPE, where every value is incremented by 1 to handle links with 0 clicks). We compare our model to a real-time interpolation state-of-the-art method to predict views on YouTube [23]. This method uses a Hawkes Intensity Process (HIP) to model the aggregate impact and decay from multiple shares. The interpolated number of clicks is the sum of the influence of current shares and the sum of influences of deprecated previous shares, not unlike our equation. However, HIP leverages 6 parameters capturing several other features like the content type, the network of diffusion, and content sensitivity to promotion. We reproduced HIP, using two training periods for comparison - hour 1 and hours 1-to-4 of the lifespan of the post. HIP trains using shares (retweets) and clicks, while our model follows a different evolution model and uses receptions, impressions and clicks.

Geometric decay is more robust A quick glance at the comparative interpolation results (Table 2 and Figure 6) highlight the merit of a geometric method. We outperform the state of the art, with 5-8x smaller relative errors. The HIP model was not initially designed

\begin{tabular}{cc|ccc}
\hline Training & Method & \multicolumn{3}{|c}{ MAPE (adj.) by \# clicks } \\
& & None & $\mathbf{1 - 5}$ & $>\mathbf{5}$ \\
\hline Hour 1 & HIP & $772 \%$ & $1205 \%$ & $691 \%$ \\
Hours 1-4 & HIP & $\mathbf{3 8 . 7} \%$ & $327 \%$ & $551 \%$ \\
Other domains & Geom. & $305 \%$ & $\mathbf{3 0 1} \%$ & $\mathbf{6 6} \%$ \\
\hline
\end{tabular}

Table 3: Comparing cross domain robustness for HIP and the Geometric method in predicting 24-hour clicks, by article popularity.

to include the effect of the number of followers, so we refine this method to use receptions as a feature instead of shares. Shown as HIP (rec), we find that this improves with sufficient training, but it still falls short of our model. The scatterplot reveals that HIP, while it is initially well trained underestimate clicks that occur later in time. In contrast, our geometric evolution, which is memoryless, is more robust to the noise added with time. We further validate that result by training HIP to predict impressions instead of clicks. Once more, (results omitted due to space constraints), we find that at this time-scale, the geometric evolution returns much more accurate estimations in the case of Twitter.

Robustness across news domains Our method follows a relatively simple dynamic, but we cannot exclude that its superior accuracy originates from idiosyncrasies present in BuzzFeed. We turn to Multiple News Media to study the robustness of interpolation in other domains. A caveat to keep in mind is that for those links, we only have access to the bit. ly hourly click counts reported by these URLs which are not a perfect ground truth. In a first validation, we use a random third of the data as training and achieve MAPE of $58 \%$, arguably comparable to noise introduced by bit. ly. To further ensure that we are not dependent on content-specific features, we employed a leave-one-out strategy where the parameters $(q, s)$ were learned from the 4 other domains, and link specific CPI estimated at hour 1. Our interpolation errors remains below $100 \%$ and often approaches $50 \%$ for more than half of the links.

We compare HIP's performance on these other domains. Since HIP is designed to learn the model from the initial trajectory of shares, we train on the first hour and first four hours. In contrast, the memoryless model was trained using a leave-one-out strategy. For more popular articles, the memoryless model outperforms HIP (Figure 3).

\section{CONCLUSIONS}

This research validates the use of a publicly accessible method of analyzing social news consumption on Twitter. The analyses provide further evidence that commonly used popularity metrics such as the number of shares or posts have flaws. In our analyses, we show that the relationship between shares and clicks is not straightforward. Rather, it is a two-stage process with a key intermediate piece of information: impressions.

Using this insight, we developed a model based on this twostage process to predict the temporal dynamics of a post using publicly available data. Compared to many other models, our model only relies on the first hour of data. In particular, it only relies on easily obtainable public information. As such, it provides utility in forecasting applications with time constraints.

Overall, we hope that our model and methodology will help foster better understanding of sharing and audience dynamics. 


\section{REFERENCES}

[1] J An, Meeyoung Cha, Krishna Gummadi, and Jon Crowcroft. 2011. Media landscape in Twitter: A world of new conventions and political diversity. In Proceedings of the International Conference Weblogs and Social Media (ICWSM). 18-25.

[2] J An, D Quercia, Meeyoung Cha, Krishna Gummadi, and Jon Crowcroft. 2014 Sharing political news: the balancing act of intimacy and socialization in selective exposure. EPf Data Science (2014).

[3] S. Asur and B A Huberman. 2010. Predicting the Future with Social Media. In Web Intelligence and Intelligent Agent Technology (WI-IAT), 2010 IEEE/WIC/ACM International Conference on. 492-499.

[4] Eytan Bakshy, Jake M Hofman, Winter A Mason, and Duncan J Watts. 2011. Everyone's an influencer: quantifying influence on twitter. In WSDM '11: Proceedings of the fourth ACM international conference on Web search and data mining. ACM Request Permissions.

[5] J Bollen, H Mao, and X Zeng. 2011. Twitter mood predicts the stock market. fournal of Computational Science (2011)

[6] danah boyd, Scott Golder, and Gilad Lotan. 2010. Tweet, Tweet, Retweet: Conversational Aspects of Retweeting on Twitter. In System Sciences (HICSS), 2010 43rd Hawaii International Conference. IEEE, Honolulu, HI, 1-10.

[7] Meeyoung Cha, H Haddadi, F Benevenuto, and Krishna Gummadi. 2010. Measur ing User Influence in Twitter: The Million Follower Fallacy. In Proceedings of the International Conference Weblogs and Social Media (ICWSM).

[8] P Chebolu and P Melsted. 2008. PageRank and the random surfer model. In Proceedings of ACM-SIAM SODA.

[9] Justin Cheng, Lada A Adamic, P Alex Dow, Jon Michael Kleinberg, and Jure Leskovec. 2014. Can cascades be predicted?. In WWW'14: Proceedings of the $23 r d$ international conference on World wide web. International World Wide Web Conferences Steering Committee.

[10] Ayman Farahat and Michael C Bailey. 2012. How effective is targeted advertising? In WWW '12: Proceedings of the 21st international conference on World Wide Web. ACM Request Permissions.

[11] Maksym Gabielkov, Arthi Ramachandran, Augustin Chaintreau, and Arnaud Legout. 2016. Social Clicks: What and Who Gets Read on Twitter? SIGMETRICS '16: Proceedings of the ACM SIGMETRICS/international conference on Measurement and modeling of computer systems (June 2016).

[12] Maksym Gabielkov, Arthi Ramachandran, Arnaud Legout, and Augustin Chaintreau. 2016. Social Clicks: What and Who Gets Read on Twitter?. In ACM SIGMETRICS / IFIP Performance 2016. Antibes Juan-les-Pins, France.

[13] A B Hubert, T Hubert, and C Mugizi. 2006. A Random-Surfer Web-Graph Model. In Proc. of ANALCO'06. New York, USA.

[14] Eric Jones, Travis Oliphant, Pearu Peterson, and others. 2001. SciPy: Open source scientific tools for Python. Technical Report.

[15] Yaser Keneshloo, Shuguang Wang, Eui Hong Han, and Naren Ramakrishnan 2016. Predicting the popularity of news articles. In 16th SIAM International Conference on Data Mining 2016, SDM 2016. Virginia Polytechnic Institute and State University, Blacksburg, United States, 441-449.

[16] Haewoon Kwak, Changhyun Lee, Hosung Park, and Sue Moon. 2010. What is Twitter, a social network or a news media?. In WWW'10: Proceedings of the 19th international conference on World wide web. ACM.

[17] D C Liu and J Nocedal. 1989. On the Limited Memory BFGS Method for Large Scale Optimization. Math. Program. 45, 3 (Dec. 1989), 503-528.
[18] Travis Martin, Jake M Hofman, Amit Sharma, Ashton Anderson, and Duncan J Watts. 2016. Exploring Limits to Prediction in Complex Social Systems. In Proceedings of the 25th International Conference on World Wide Web. International World Wide Web Conferences Steering Committee, Republic and Canton of Geneva, Switzerland, 683-694.

[19] H B McMahan, G Holt, D Sculley, M Young, and D Ebner. 2013. Ad Click Prediction: a View from the Trenches. KDD '16: Proceedings of the 22th ACM SIGKDD international conference on Knowledge discovery and data mining (2013).

[20] SA Myers, C Zhu, and Jure Leskovec. 2012. Information Diffusion and External Influence in Networks. KDD '12: Proceedings of the 18th ACM SIGKDD international conference on Knowledge discovery and data mining (2012). arXiv:5B22CD56A65B-43CA-95AB-4E21D8FE35A6

[21] Henrique Pinto, Jussara M Almeida, and Marcos A Gonçalves. 2013. Using early view patterns to predict the popularity of youtube videos. ACM, New York, New York, USA.

[22] Matthew Richardson, Ewa Dominowska, and Robert Ragno. 2007. Predicting clicks: estimating the click-through rate for new ads. In WWW'07: Proceedings of the 16th international conference on World Wide Web. ACM.

[23] Marian-Andrei Rizoiu, Lexing Xie, Scott Sanner, Manuel Cebrian, Honglin Yu, and Pascal Van Hentenryck. 2017. Expecting to Be HIP: Hawkes Intensity Processes for Social Media Popularity. In Proceedings of the 26th International Conference on World Wide Web. International World Wide Web Conferences Steering Committee, Republic and Canton of Geneva, Switzerland, 735-744.

[24] Adam Sadilek and Henry Kautz. 2013. Modeling the impact of lifestyle on health at scale. In WSDM '13: Proceedings of the sixth ACM international conference on Web search and data mining. ACM Request Permissions.

[25] Hua-Wei Shen, Dashun Wang, Chaoming Song, and Albert-László Barabasi. 2014. Modeling and Predicting Popularity Dynamics via Reinforced Poisson Processes. available on arXiv.org (Jan. 2014). arXiv:1401.0778v1

[26] Gabor Szabo and Bernardo A Huberman. 2010. Predicting the popularity of online content. Commun. ACM 53, 8 (Aug. 2010).

[27] Lucy Wang, Arthi Ramachandran, and Augustin Chaintreau. 2016. Measuring Click and Share Dynamics on Social Media: A Reproducible and Validated Approach. Proceedings of AAAI ICWSM Workshop on News and Public Opinion (May 2016).

[28] Xiaofeng Wang, Matthew S Gerber, and Donald E Brown. 2012. Automatic crime prediction using events extracted from twitter posts. In SBP'12: Proceedings of the 5th international conference on Social Computing, Behavioral-Cultural Modeling and Prediction. Springer-Verlag.

[29] FMF Wong, S Sen, and M Chiang. 2012. Why watching movie tweets won't tell the whole story?. In Proceedings of the 2012 ACM workshop....

[30] Tauhid Zaman, Emily B Fox, and Eric T Bradlow. 2014. A Bayesian Approach for Predicting the Popularity of Tweets. Annals of Applied Statistics 8, 3 (Sept. 2014), 1583-1611.

[31] Qingyuan Zhao, Murat A Erdogdu, Hera Y He, Anand Rajaraman, and Jure Leskovec. 2015. SEISMIC. In the 21th ACM SIGKDD International Conference. ACM Press, New York, New York, USA, 1513-1522.

[32] Oingyuan Zhao, Murat A Erdogdu, Hera Y He, Anand Rajaraman, and Jure Leskovec. 2015. SEISMIC: A Self-Exciting Point Process Model for Predicting Tweet Popularity. available on arXiv.org (June 2015). arXiv:1506.02594v1 